ORIGINAL

\title{
Aflatoxicosis en chinchillas (Chinchilla lanigera)
}

\section{Aflatoxicosis in chinchillas (Chinchilla lanigera)}

\author{
Sandra Cepeda A, ${ }^{1}$ Q.A., Hansen Murcia G, ${ }^{1}$ M.Sc., Gonzalo Diaz G, ${ }^{1 *}$ Ph.D. \\ ${ }^{1}$ Universidad Nacional de Colombia, Facultad de Medicina Veterinaria y Zootecnia, Laboratorio \\ de Toxicología, Bogotá, Colombia. *Correspondencia: gjdiazg@unal.edu.co
}

Recibido: Marzo de 2010; Aceptado: Diciembre de 2010.

\section{RESUMEN}

Objetivo. Determinar los efectos de las aflatoxinas (AF) en chinchillas (Chinchilla lanigera) por consumo de dietas contaminadas a diferentes concentraciones. Materiales y métodos. Un total de 20 chinchillas macho se distribuyeron en 5 grupos experimentales de 4 animales cada uno, los cuales recibieron diferentes niveles de aflatoxinas totales en el alimento durante 8 semanas: 0, 25, 50, 100 y 200 ppb. Semanalmente se determinó el consumo de alimento, el peso corporal individual y la mortalidad. Adicionalmente, al finalizar el experimento, se investigó el metabolismo hepático in vitro de la aflatoxina B1 (AFB1) en animales no expuestos al tóxico y se realizó examen histopatológico del hígado en todos los grupos. Resultados. El consumo de dietas contaminadas con aflatoxinas no causó efectos sobre el consumo de alimento ni hubo mortalidad. Solamente se observó disminución significativa $(p<0.05)$ en la ganancia de peso corporal en los animales expuestos a $200 \mathrm{ppb}$ en la dieta. Los niveles de 100 y 200 ppb (ng/g) de aflatoxinas indujeron cambios leves a moderados en el hígado. El metabolismo microsomal de la AFB1 demostró la producción del epóxido de la AFB1 como único metabolito. Conclusiones. Los resultados del presente estudio demuestran que la chinchilla es altamente resistente a las aflatoxinas, pudiendo tolerar durante al menos dos meses niveles de aflatoxinas en el alimento que resultarían letales, en apenas unos pocos días, para especies sensibles como el cerdo o el perro.

Palabras clave: Chinchilla, aflatoxinas, aflatoxicosis, metabolismo, in vitro. (Fuente: AIMS) 


\section{ABSTRACT}

Objetive. To determine the effects of dietary aflatoxins (AF) on chinchillas (Chinchilla lanigera). Materials and methods. A total of 20 male chinchillas were distributed in 5 experimental groups of 4 animals each. Animals received the following levels of dietary AF: $0,25,50,100$ and $200 \mathrm{ppb}(\mathrm{ng} / \mathrm{g})$ for 8 weeks. Feed intake, individual body weight and mortality were recorded weekly. In addition, the in vitro hepatic metabolism of aflatoxin B1 (AFB1) was investigated using liver samples from the control group. Results. Exposure to AF did not cause any effect on feed intake or mortality. The only parameter affected significantly $(\mathrm{p}<0.05)$ was body weight when $200 \mathrm{ppb}$ AF were added to the diet. Both AF levels of 100 and $200 \mathrm{ppb}$ produced mild to moderate liver changes. Microsomal metabolism of AFB1 showed production of AFB1 epoxide as the only biotransformation product. Conclusions. Results demonstrated that chinchillas were highly resistant to AF and were capable of tolerating for at least two months dietary aflatoxin levels that would be lethal, in just a few days, to sensitive species such as pigs or dogs, in just a few days.

Key words: Chinchilla, aflatoxins, aflatoxicosis, metabolism, in vitro. (Source: AIMS)

\section{INTRODUCCIÓN}

La chinchilla (Chinchilla lanigera) es una especie de roedor que habitaba naturalmente en los Andes de Suramérica, a altitudes comprendidas entre 3.000 y 5.000 metros sobre el nivel del mar. La calidad de su pelaje y su gran valor comercial llevó a la caza excesiva acabando prácticamente con las poblaciones silvestres (1). Actualmente existen en granjas de producción, especialmente en países como Argentina, Chile y Bolivia. En Colombia existen algunos criaderos que han tomado la producción de esta especie como una alternativa económica debido a las ventajas competitivas de la producción de piel y su potencial de comercialización en el exterior. En varios de estos criaderos localizados en la Sabana de Bogotá se presentó una alta mortalidad que fue inicialmente atribuida a la presencia de cerca de $50 \mathrm{ppb}(\mathrm{ng} / \mathrm{g})$ de aflatoxinas (AF) totales en el alimento (caso clínico de los autores, sin publicar).

Las AF son un grupo de metabolitos heterocíclicos sintetizados por algunas cepas de los hongos Aspergillus flavus y Aspergillus parasiticus. De forma natural estos hongos pueden producir cuatro tipos de AF conocidos como aflatoxinas B1 (AFB1), B2 (AFB2), G1 (AFG1) y G2 (AFG2) (2). En la mayoría de las especies de mamíferos la AFB1 se biotransforma a una forma tóxica del compuesto conocida como aflatoxina- 8,9-epóxido (AFBO) por la acción de enzimas de la familia citocromo P450 (3). Este metabolito es capaz de unirse a los residuos de guanina de los ácidos nucleicos causando alteraciones irreversibles que pueden llevar a carcinogenicidad, mutagenicidad y teratogenicidad (4-7). En animales de producción, además de los efectos carcinógenos, se pueden presentar reducción en la ganancia en peso, efectos adversos en la reproducción y en el sistema inmunológico e incluso la muerte si la dosis de la micotoxina es suficientemente alta $(8,9)$. En cuanto al efecto de las $A F$ en los roedores se ha encontrado que existe una gran variabilidad en la sensibilidad dependiendo de la especie. Por ejemplo, el género Mastomys spp. (un roedor africano) es altamente resistente a las AF mientras que el ratón y el hámster presentan una susceptibilidad intermedia, en contraste, la rata es altamente sensible $(3,10,11)$.

Debido a la sensibilidad diferencial entre roedores a las AF y a que no existen estudios sobre la sensibilidad de las chinchillas a las AF es importante determinar los efectos de estas micotoxinas en esta especie de roedor. El objetivo del presente estudio fue investigar los efectos de diferentes niveles de AF en el alimento en la especie Chinchilla lanigera. Adicionalmente se investigó el metabolismo hepático in vitro 
de la AFB1 en esta misma especie con el fin de determinar el perfil de metabolitos fase I producidos a partir esta aflatoxina.

\section{MATERIALES Y MÉTODOS}

Aflatoxinas. Las aflatoxinas utilizadas como fuente de contaminación del alimento fueron producidas a partir de la cepa de Aspergillus parasiticus R39 aislada en Colombia a partir de una muestra de maíz en el Laboratorio de Toxicología de la Facultad de Medicina Veterinaria y Zootecnia de la Universidad Nacional de Colombia. Esta cepa se encuentra depositada en el cepario de la Universidad de los Andes (Bogotá, Colombia).

Para la propagación del hongo toxigénico y producción de la micotoxina se utilizó arroz comercial como sustrato (12). El arroz se mezcló con agua en proporción $1: 2$ y se llevó a cocción durante 30 minutos. Posteriormente se pesaron $200 \mathrm{~g}$ del arroz cocido a los que se agregaron $125 \mathrm{~mL}$ de agua que se mezclaron hasta obtener una pasta homogénea. De esta pasta se pesaron $40 \mathrm{~g}$ en un Erlenmeyer, se agregaron 10 $\mathrm{mL}$ de agua destilada, se esterilizó en autoclave a $121^{\circ} \mathrm{C}$ y 15 pulgadas/cm2 de presión durante 15 minutos y una vez a temperatura ambiente se inoculó con $1 \mathrm{~mL}$ de una dilución de $60 \times 104$ conidias de la cepa R39. En total se prepararon de esta manera un total de 30 Erlenmeyers que se sometieron a incubación a $27^{\circ} \mathrm{C}$ durante 28 días en ausencia de luz.

\section{Extracción de las aflatoxinas y} cuantificación. La extracción de las aflatoxinas se llevó a cabo de acuerdo con lo descrito en estudios previos (13). El contenido de cada Erlenmeyer se envolvió en papel filtro cualitativo y se llevó a un extractor tipo Soxhlet. La extracción en Soxhlet se realizó a $55^{\circ} \mathrm{C}$ por $24 \mathrm{~h}$ utilizando metanol como solvente. Posteriormente el solvente fue removido utilizando un evaporador rotario a una temperatura de $55^{\circ} \mathrm{C}$. Al residuo seco se adicionó una mezcla de cloroformo y agua $(2: 1, \mathrm{v} / \mathrm{v})$ y se dejó en reposo hasta observar la separación de las fases acuosa y orgánica. La fase orgánica se retiró cuidadosamente y se llevó a sequedad a $40^{\circ} \mathrm{C}$ para disolverla nuevamente con una solución de metanol, agua y éter de petróleo en proporción 10:1:10. De nuevo se retiró la fase orgánica y se llevó a sequedad. Finalmente las toxinas se reconstituyeron en $10 \mathrm{~mL}$ de una mezcla 84:16 de acetonitrilo y agua.

El extracto obtenido se diluyó 1:250.000 en agua, se homogenizó y una alícuota del mismo se utilizó para cuantificar la concentración de aflatoxinas por cromatografía líquida de alta eficiencia de acuerdo con lo descrito por Diaz et al (14).

Ensayo in vivo. Para evaluar los efectos de las AF en las chinchillas se utilizaron un total de 20 animales machos de entre 6 y 11 semanas de edad, los cuales se distribuyeron aleatoriamente en 5 grupos experimentales de 4 animales cada uno de manera que no se presentaran diferencias significativas en el peso corporal promedio entre los diferentes grupos. Los animales se alojaron de manera individual en jaulas de acero y se les suministró agua y alimento para conejos ad libitum (este tipo de alimento es comúnmente utilizado en explotaciones comerciales de chinchillas debido a que no existe alimento comercial para esta especie de lagomorfo).

El alimento utilizado no presentaba niveles detectables de aflatoxinas. Los cinco tratamientos experimentales correspondieron a diferentes niveles de aflatoxinas totales en la dieta, así: grupo 1 (control), sin adición de aflatoxinas; grupo 2: 25 ppb; grupo 3: 50 ppb; grupo 4: 100 ppb y grupo 5: $200 \mathrm{ppb}$. Las aflatoxinas fueron disueltas en acetona, adicionadas a las dietas en harina y homogenizadas tanto manualmente como en una mezcladora de palas. Posteriormente las dietas fueron peletizadas y suministradas durante 8 semanas, al término de las cuales los animales fueron sacrificados con el fin de tomar muestras de tejido hepático para análisis histopatológico. Durante el transcurso del experimento se determinó el consumo de alimento y el peso corporal de forma semanal. Los animales experimentales fueron tratados de manera ética, brindando el mayor bienestar posible durante el ensayo. 
Metabolismo hepático in vitro de la aflatoxina B1. Para determinar el metabolismo hepático in vitro de la AFB1 se obtuvieron microsomas de los hígados de los animales del grupo control de acuerdo con la metodología descrita anteriormente (15). Los hígados se extrajeron inmediatamente después del sacrificio, se lavaron con PBS (fosfato $20 \mathrm{mM}, \mathrm{pH}$ 7.4, $\mathrm{NaCl} 100 \mathrm{mM}$ ) y se almacenaron a $-70^{\circ} \mathrm{C}$ hasta el momento de la extracción de los microsomas. Para la obtención de los microsomas las muestras de hígado se descongelaron parcialmente, se lavaron con PBS frío y se secaron suavemente con papel absorbente. Se pesaron aproximadamente $5 \mathrm{~g}$ en tubos de centrífuga mantenidos en hielo y se adicionaron $10 \mathrm{~mL}$ de buffer de extracción (PBS, EDTA disódico $1 \mathrm{mM}$, sacarosa $250 \mathrm{mM}$ ). Se homogenizó por 30 segundos en un homogenizador de tejidos (IKA Ultra-Turrax, Staufen, Alemania) y se centrifugó a $4^{\circ} \mathrm{C}$ por 30 minutos a $10000 \times \mathrm{g}$. Posteriormente se transfirió el sobrenadante (aproximadamente $10 \mathrm{~mL}$ ) a un tubo de ultracentrífuga mantenido en hielo y se centrifugó por 90 minutos a $98000 \times \mathrm{g}$.

Los sobrenadantes se separaron en alícuotas para ensayos posteriores y a cada pellet se adicionaron $3 \mathrm{~mL}$ de buffer de almacenamiento (buffer fosfato $20 \mathrm{mM}$ $\mathrm{pH} 7.4$, EDTA disódico $1 \mathrm{mM}$, sacarosa 250 mM, glicerol 20\%). El pellet se desprendió cuidadosamente de las paredes del tubo con una espátula delgada y se transfirió a un homogenizador de tejidos manual para resuspenderlo completamente. La suspensión microsomal se fraccionó en tubos de microcentrífuga que se almacenaron a $-70^{\circ} \mathrm{C}$ hasta su posterior utilización. Una alícuota se utilizó para determinar el contenido de proteína usando el método del ácido bicinchonínico (16) adaptado para microplaca (17).

La determinación de la actividad enzimática de los microsomas se realizó en tubos de microcentrífuga de $1.5 \mathrm{~mL}$ que contenían glucosa 6-fosfato $5 \mathrm{mM}, \mathrm{NADP}+0.5 \mathrm{mM}$, glucosa 6-fosfato deshidrogenasa 0.5 UI, 2 $\mu \mathrm{L}$ de sustrato (AFB1) en DMSO equivalente al $0.8 \%$ del solvente de incubación (18),
$100 \mu \mathrm{g}$ de proteína microsomal y buffer de

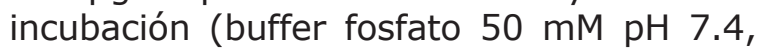
$\mathrm{MgCl} 5 \mathrm{mM}$, EDTA $0.5 \mathrm{mM}$ ) hasta un volumen de $250 \mu \mathrm{L}$. Las incubaciones se llevaron a cabo durante 10 minutos a $39^{\circ} \mathrm{C}$ luego de los cuales se adicionaron $250 \mu \mathrm{L}$ de acetonitrilo a $-20^{\circ} \mathrm{C}$ para detener la reacción (19). El contenido del vial se homogenizó y centrifugó a 12.000 x g por 10 minutos y se analizaron $50 \mu \mathrm{L}$ por HPLC según la metodología descrita por Lozano y Diaz (15). Debido a la inestabilidad de la forma epóxido de la AFB1 y su rápida hidrólisis a una forma más estable conocida como AFB1-dihidrodiol (dhd-AFB1), el epóxido formado se cuantificó a través de la medición del dhd-AFB1 presente en la incubación. Debido a que el dhd-AFB1 no se encuentra disponible comercialmente, la cuantificación de este se realizó utilizando como estándar aflatoxina B2 $\alpha$ (AFB2 $\alpha$ ) compuesto que presenta características fluorométricas y espectrales equivalentes (20).

Análisis estadístico. El consumo de alimento y la ganancia de peso corporal de los diferentes tratamientos experimentales se analizaron mediante ANOVA y contrastes ortogonales contra el grupo control utilizando la prueba de Dunnett cuando el ANOVA mostró diferencias significativas $(p<0.05)$.

\section{RESULTADOS}

La inoculación del medio de arroz con la cepa R37 de Aspergillus parasiticus resultó en la producción de las cuatro aflatoxinas de ocurrencia natural en la proporción 62.9:9.58:26.3:1.20 para las aflatoxinas B1, B2, G1 y G2, respectivamente. La concentración de aflatoxinas totales en el extracto final fue de $1.67 \mathrm{~g} / \mathrm{l}(1.05 \mathrm{~g} / \mathrm{l}$ de AFB1, $0.16 \mathrm{~g} / \mathrm{l}$ de AFB2, $0.44 \mathrm{~g} / \mathrm{l}$ de AFG1 y $0.02 \mathrm{~g} / \mathrm{l}$ de AFG2). La producción de estos niveles de aflatoxinas permitió contaminar la dieta control para obtener las dietas experimentales a concentraciones de 25, 50,100 y $200 \mathrm{ppb}(\mu \mathrm{g} / \mathrm{kg})$.

No se presentaron diferencias significativas en el consumo de alimento semanal o acumulado entre los diferentes grupos 
experimentales. Tampoco se observó mortalidad durante las 8 semanas que duró el experimento. La ganancia de peso en el grupo control fue de $14.6 \mathrm{~g} / \mathrm{semana}$, en el grupo que recibió $25 \mathrm{ppb}$ de AF totales fue de $13.5 \mathrm{~g} /$ semana y en el grupo alimentado con $50 \mathrm{ppb}$ fue de $11.8 \mathrm{~g} / \mathrm{semana}$. Con niveles de 100 y 200 ppb en la dieta la ganancia de peso promedio fue de de 9.9 y $8.8 \mathrm{~g} /$ semana, respectivamente. A pesar de las diferencias numéricas en la ganancia de peso observadas entre el grupo control y los grupos que recibieron 50, 100 y 200 ppb de AF totales, solamente se presentó una diferencia significativa con el grupo que recibió 200 ppb con relación al control. La figura 1 muestra la ganancia de peso acumulada durante las 8 semanas que duró el tratamiento experimental.

A la necropsia los hígados de las chinchillas expuestas a los niveles más altos de aflatoxinas (100 y 200 ppb) presentaron cambios consistentes en apariencia de nuez moscada y una coloración amarillenta difusa, más clara que lo normal. La evaluación histológica del hígado demostró alteraciones en los hepatocitos, principalmente vacuolización, siendo esta más severa en aquellos individuos que recibieron el alimento con 100 y 200 ppb de AF totales (Figura 2).

El estudio del metabolismo hepático de AFB1 reveló la producción de AFBO como único producto de biotransformación (Figura 3). No se detectó la producción de metabolitos tales como la aflatoxinas M1, $\mathrm{B} 2 \alpha, \mathrm{Q} 1$ o P1.

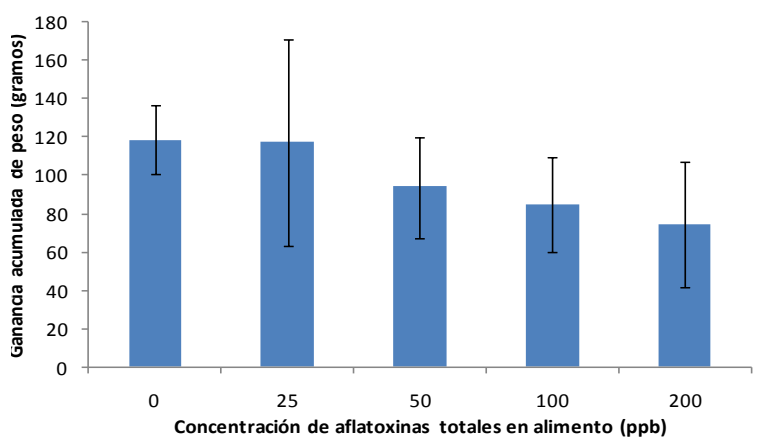

Figura 1. Ganancia de peso promedio acumulada \pm desviación estándar en chinchillas macho alimentadas 8 semanas con dietas que contenían niveles crecientes de aflatoxinas.

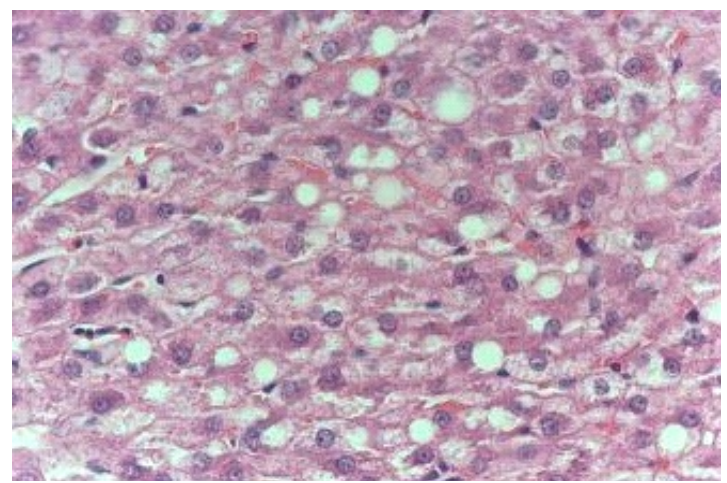

Figura 2. Microfotografía del hígado de una chinchilla macho que recibió una dieta contaminada con $200 \mathrm{ppb}$ de aflatoxinas totales en el alimento durante 8 semanas $(40 x)$. Se observa vacuolización moderada de hepatocitos.

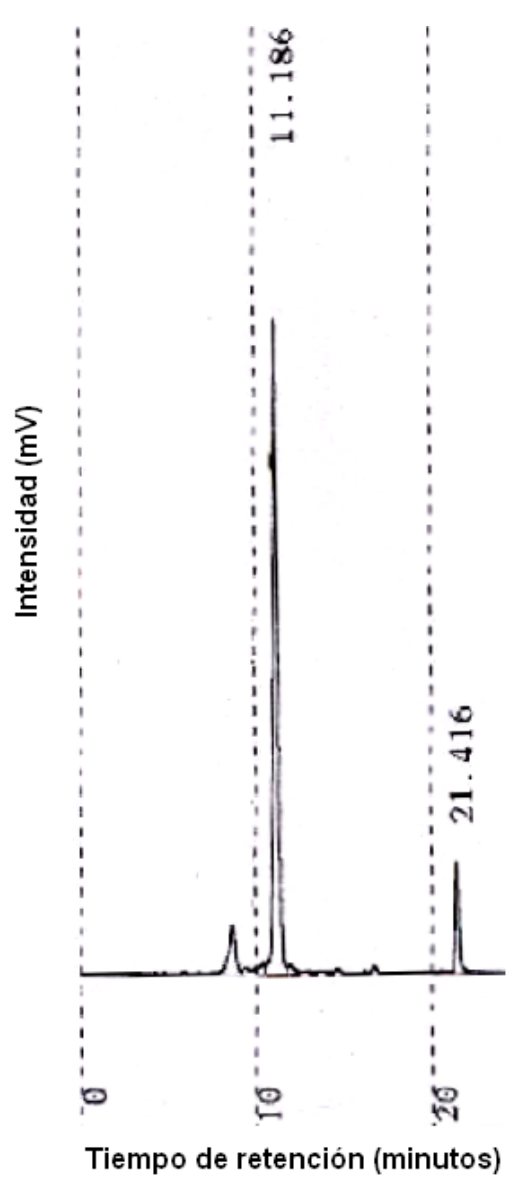

Figura 3. Cromatograma del sobrenadante de una incubación in vitro de microsomas hepáticos de chinchilla y AFB1. Se observan los picos correspondientes al sustrato (21.4 $\mathrm{min})$ y al único producto detectado correspondiente al epóxido de la AFB1 determinado como el dihidrodiol (11.1 min). 


\section{DISCUSIÓN}

Los resultados del presente estudio demostraron que las chinchillas son altamente resistentes a los efectos de las aflatoxinas, ya que a pesar de que se utilizaron niveles muy altos de aflatoxinas (hasta $200 \mathrm{ppb}$ ) durante un período de tiempo prolongado ( 8 semanas) no se presentó mortalidad y los únicos cambios observados fueron una disminución en la ganancia de peso acumulada y lesiones hepáticas de leves a moderadas. Este nivel de contaminación en el alimento resulta letal para especies sensibles como el pato y el pavo (9).

No existen estudios toxicológicos de aflatoxicosis en chinchillas que permitan contrastar los resultados del presente experimento con estudios previos. Sin embargo, recientemente se reportó un caso en el cual se atribuyó a un alimento contaminado con $212 \mathrm{ppb}$ de aflatoxinas una mortalidad aguda del $100 \%$ en 200 animales expuestos a este alimento en una granja en Argentina (1). A la luz de los resultados obtenidos en el presente estudio, es poco probable que este episodio de mortalidad aguda haya sido causado por aflatoxinas ya que en el presente estudio la exposición a 200 ppb durante 8 semanas no causó mortalidad. Es posible que la dieta de estas chinchillas haya estado contaminada con otras sustancias hepatotóxicas capaces de inducir alta mortalidad de manera aguda pero los resultados obtenidos en el presente estudio indicaron que estos efectos no se observan con 200 ppb de aflatoxinas.

Los estudios de biotransformación hepática in vitro de la AFB1 demostraron que las chinchillas biotransforman la toxina a la forma tóxica, el AFBO (detectado en su forma dhd-AFB1). El AFBO se produce en el hígado y debido a su inestabilidad y corta vida afecta principalmente este órgano causando cambio graso, aumento en la vacuolización y necrosis de hepatocitos. No se encontraron otros productos de biotransformación de la AFB1 a diferencia de lo que sucede en otras especies como rata en la que se ha reportado la producción de AFM1 y Q1 (21). El hecho de que la AFB1 sea biotransformada a AFBO pero que no se presenten efectos severos de la AFB1 en las chinchillas sugiere que la biotransformación ocurre de manera muy lenta o que el metabolismo fase II (conjugación con glutatión u otros compuestos) es el responsable de la gran resistencia de estos animales a las aflatoxinas (3). Es necesario profundizar en el estudio del metabolismo fase I y fase II de las aflatoxinas en chinchillas con el fin de dilucidar los mecanismos bioquímicos de resistencia a las aflatoxinas en esta especie.

En conclusión, Chinchilla lanigera demostró ser un roedor con una elevada resistencia a la exposición subcrónica a AF. Los únicos efectos observados tras la exposición a concentraciones de 200 ppb de AF totales en la dieta durante 8 semanas fue disminución en la ganancia de peso y alteraciones hepáticas leves a moderadas. Para determinar la causa de la elevada resistencia de las chinchillas a las AF es necesario profundizar en el metabolismo fase I determinando los parámetros enzimáticos KM y Vmax de las CYP450 responsables de su metabolismo así como también caracterizar el metabolismo fase II de detoxificación, particularmente en lo que respecta a las enzimas glutatión transferasas (GSTs), lo que se ha demostrado que estas enzimas juegan un papel clave en la resistencia a las aflatoxinas en especies como Mastomys sp., el hámster y el ratón (3) y esta podría ser también la razón de la alta resistencia a las AF observada en chinchillas. 


\section{REFERENCIAS}

1. González Pereyra ML, Carvalho ECQ, Tissera JL, Keller KM, Magnoli CE, et al. An outbreak of acute aflatoxicosis on chinchilla (Chinchilla lanigera) farm in Argentina. J Vet Diagn Invest 2008; 20:853-6.

2. Díaz GJ, Lozano MC, Acuña A. Prevalence of Aspergillus species on selected Colombian animal feedstuffs and ability of Aspergillus section Flavi to produce aflatoxins. World Mycot J 2009; 2:31-4.

3. Esaki H, Kumagai S. Glutathione-Stransferase activity toward aflatoxin epoxide in livers of mastomys and other rodents. Toxicon 2002.

4. Do JH, Choi D. Aflatoxins: detection, toxicity, and biosynthesis. Biotechnol Bioprocess Eng 2007; 12:585-93.

5. Eaton DL, Gallagher EP. Mechanisms of aflatoxin carcinogenesis. Annu Rev Pharmacol Toxicol 1994; 34:135-72.

6. Ferguson LR, Philpott M. Nutrition and mutagenesis. Annu Rev Nutr 1994; 28:313-29.

7. Verma RJ. Aflatoxin causes DNA damage. Int J Hum Genet 2004; 4:231-6.

8. Hussein HS, Brasel JM. Toxicity, metabolism, and impact of mycotoxins on humans and animals. Toxicology $2001 ; 167: 101-34$.

9. Leeson S, Diaz GJ, Summers JD. Poultry metabolic disorders and mycotoxins. Guelph (ON): University Books; 1995.

10. Tulayakul P, Sakuda S, Dong KS, Kumagai S. Comparative activities of glutathione-S-transferase dialdehyde reductasa toward aflatoxin B1 in livers of experimental and farm animals. Toxicon 2005; 46:204-9.
11. Kumagai $S$, Sugita-Konishi $Y$, HaraKudo Y, Ito Y, Nogushi Y, Yamamoto Y, Ogura $A$. The fate and acute toxicity of aflatoxin B1 in the mastomys and rat. Toxicon 1998; 36:179-88.

12. Shotwell, OL, Hesseltine CW, Stubblefield RD, Soreson WG. Production of aflatoxin on rice. Appl Microbiol 1966; 425-428.

13. Sargeant K, Carnaghan RBA, Allcroft $\mathrm{R}$. Toxic products in groundnuts. Chemistry and origin. Chem Ind 1963; 53-55.

14. Diaz GJ, Perilla NS, Rojas Y. Occurrence of aflatoxins in selected Colombian foods. Mycotox Res 2001; 17:15-9.

15. Lozano MC, Diaz GJ. Microsomal and cytosolic biotransformation of aflatoxin B1 in four poultry species. Br Poult Sci 2006; 734-41.

16. Smith PK, Krohn RI, Hermanson GT, Mallia AK, Gartner FH, Provenzano, M.D. et al. Measurement of protein using bicinchoninic acid. Anal Biochem $1985 ; 150: 76-85$.

17. Redinbaugh MG, Turley RB. Adaptation of the bicinchoninic acid protein assay for the use with microtiter plates and sucrose gradient fractions. Anal Biochem 1986; 153:267-71.

18. Busby WF, Ackermann JM, Crespi CL. Effect of methanol, ethanol, dimethyl sulphoxide and acetonitrile on in vitro activities of cDNA-expressed human cytochromes P-450. Drug Metab Dispos 1999; 27:246-9.

19. Blanchard J. Evaluation of the relative efficacy of various techniques for deproteinizing plasma samples prior to high-performance liquid chromatographic analysis. J Chromatogr 1981; 226:455-60. 
20. Neal GE, Judah DJ, Stirpe F, Patterson DSP. The formation of 2,3-dihydroxy2,3-dihydro-aflatoxin $B 1$ by the metabolism of aflatoxin B1 in liver microsomes isolated from certain avian and mammalian species and the possible role of this metabolite in the acute toxicity of aflatoxin B1. Toxicol Appl Pharmacol 1981; 58:431-7.
21. Neal, G.E., Colley, P.J. Some highperformance liquid-chromatoghraphy studies of the metabolism of aflatoxins by rat liver microsomal preparations. Biochem J 1978; 174:839-51. 